\title{
More on gravitational memory
}

\author{
Pujian $\mathbf{M a o}^{a, c}$ and Xiaoning $\mathbf{W} \mathbf{u}^{b, d}$ \\ ${ }^{a}$ Center for Joint Quantum Studies and Department of Physics, School of Science, \\ Tianjin University, \\ 135 Yaguan Road, Tianjin 300350, P.R. China \\ ${ }^{b}$ Institute of Mathematics, Academy of Mathematics and System Science, \\ Chinese Academy of Sciences, \\ Beijing 100190, P.R. China \\ ${ }^{c}$ Institute of High Energy Physics and Theoretical Physics Center for Science Facilities, \\ Chinese Academy of Sciences, \\ 19B Yuquan Road, Beijing 100049, P.R. China \\ ${ }^{d}$ Hua Loo-Keng Key Laboratory of Mathematics, Chinese Academy of Sciences, \\ Beijing 100190, China \\ E-mail: pjmao@tju.edu.cn, wuxn@amss.ac.cn
}

ABSTRACT: Two novel results for the gravitational memory effect are presented in this paper. We first extend the formula for the memory effect to solutions with arbitrary two surface boundary topology. The memory effect for the Robinson-Trautman solution is obtained in its standard form. Then we propose a new observational effect for the spin memory. It is a time delay of time-like free falling observers.

Keywords: Classical Theories of Gravity, Gauge Symmetry

ArXiv EPrint: 1812.07168 


\section{Contents}

1 Introduction 1

2 Displacement memory effect $\quad 2$

3 Robinson-Trautman waves 4

4 Spin memory effect 5

5 Conjugate points $\quad 8$

6 Discussions $\quad 9$

$\begin{array}{ll}\text { A Newman-Unti solution in the Newman-Penrose formalism } & 10\end{array}$

B Weyl transformation of solutions $\quad 12$

\section{Introduction}

The gravitational memory effect was first reported by Zel'dovich and Polnarev [1] in linearized gravity and further investigated by Christodoulou in full Einstein gravity [2] (see also [3-7] for further development and [8-10] for the realization with gravitational wave detectors). The memory effect is a relative displacement of nearby observers. It is therefore called the displacement memory effect. Recently, memory effects have obtained renewed interest from a purely theoretical point of view. A fundamental connection between the displacement gravitational memory effect and Weinberg's soft graviton theorem [11] was discovered by Strominger and Zhiboedov [12]. ${ }^{1}$ The gravitational memory formula and the Fourier transformation of Weinberg's soft graviton formula are mathematically equivalent.

A recent investigation on soft graviton theorems [21] shows that the universal property goes beyond Weinberg's pole formula and contains next-to-leading orders in the low-energy expansion. Inspired by the sub-leading soft graviton theorem, a new gravitational memory was proposed in [22]. This new gravitational memory effect is suggested to be a relative time delay between different orbiting light rays induced by radiative angular momentum flux. Accordingly it is called the spin memory.

The standard treatment of the memory effect $[2,7]$ is based on a special choice of the topology of null infinity which is $S^{2} \times \mathbb{R}$. From the geometrical point of view, null infinity is not part of space-time but can be added to it by conformal compactification [23, 24]. Hence, the topology of any asymptotically flat space-time can be always set to be the

\footnotetext{
${ }^{1}$ See also the analogue in gauge theory $[13-20]$.
} 
standard $S^{2} \times \mathbb{R}$ via changing the conformal factor during the compactification. However, asymptotically flat solutions may not be in their simplest form with the boundary topology being unit 2 sphere but an arbitrary 2 surface. Relating those solutions to the standard boundary topology will lose the simplicity both geometrically and algebraically. For instance, the well-known Robinson-Trautman waves [25] will not be truncated in the $\frac{1}{r}$ expansion with a unit 2 sphere boundary topology. It is extremely difficult, if not impossible, to see the geometrical property of this simple but very important exact solution with gravitational radiation. Therefore, it is definitely meaningful to extend the formula of the memory effect to the case of arbitrary 2 surface boundary topology. This is precisely what we will show in following pages.

Another purpose of this paper is to provide a new observational effect of the spin memory. In [22], it is proposed that light rays orbiting in different directions acquire a relative delay which will induce a shift in the interference fringe. Alternatively, we propose to examine the spin memory effect by time-like free falling observers who are very close to null infinity. We find that a free falling observer, which is initially static, is forced to orbit by the gravitational radiation (see also [26-33]). It receives a time delay due to massive objects in the space-time, e.g massive stars or black holes, and gravitational radiation (see also [34]). The former is the well-known Shapiro time delay [35] (see [36, 37] for recent development) and was observationally verified almost 40 years ago [38], while the latter is less stressed elsewhere. If a ring of freely falling observers who are initially static and synchronized can be set, the changes of the proper time of every observer on this ring will be different at later time. It can therefore memorize the waveform of the gravitational waves. Though a stationary massive object can cause a time delay for a single observer, the change in proper time of each observer is the same. Consequently, the ring of free falling observers will detect two memory effects: the displacement memory that will squash and stretch the shape of the ring and the time delay that will cause a difference in the proper time of nearby observers.

The plan of this paper is quite simple. In the next section, we will derive the formula of the displacement memory with arbitrary 2 surface boundary topology. Section 3 will present the displacement memory of Robinson-Trautman waves as a precise example. In section 4 , we compute the time delay formula of free falling time-like observers. Then we will prove that conjugate points on a time-like geodesic are very far from each other in the asymptotic region in section 5 . Some comments will be given in the discussion section. There are also two appendices providing useful information for the main text.

\section{Displacement memory effect}

By setting the boundary topology to be $S^{2} \times \mathbb{R}$, the null basis vector $n$ in the standard Newman-Penrose formalism [39] is tangent to null geodesics with affine parameter $u$ on null infinity. The displacement memory effect in such cases is controlled by the time integration of the asymptotic shear of $n$, i.e. $\lambda^{0}$. This is equivalent to the change of the asymptotic shear of $l$, i.e. $\sigma^{0}$, at early time $u_{i}$ and late time $u_{f}$ [7]. However, according to Newman-Unti [40] (see also appendix A), the most general asymptotically flat solution 
is derived with the boundary topology being arbitrary 2 surface including the well-known Robinson-Trautman metrics [25]. Actually, Robinson-Trautman is very special as it allows a shear-free null geodesic congruence, namely $\sigma=0$. From the standard formula, nothing will be memorized, but it is indeed an exact solution representing spherical radiation. Clearly, a general formula of displacement memory is of urgent need for answering this type of question. This is what we will setup in this section.

We will first check the memory formula for the solutions with arbitrary 2 surface null boundary topology by mapping them to the unit 2 sphere via Weyl transformations, as is well studied very recently by Barnich and Troessaert [41]. In appendix B, relevant results are presented and we will follow the convention of [41]. We use $(u, z, \bar{z})$ coordinates and unprimed quantities for the unit 2 sphere case with boundary metric $d s^{2}=\frac{4}{(1+z \bar{z})^{2}} d z d \bar{z}$ while $\left(u^{\prime}, z^{\prime}, \bar{z}^{\prime}\right)$ coordinates $^{2}$ and primed quantities are for the solution in its original form with arbitrary 2 surface boundary metric $d s^{2}=\frac{2}{P^{\prime} P^{\prime}} d z^{\prime} d \bar{z}^{\prime}$. The two coordinates are connected in the following way ${ }^{3}$

$$
z^{\prime}=z, \quad u^{\prime}=\int_{0}^{u} d v \frac{P_{s}}{\sqrt{P^{\prime} \bar{P}^{\prime}}}, \quad P_{s}=\frac{1+z \bar{z}}{\sqrt{2}} .
$$

Under such a coordinate transformation, $\sigma^{\prime 0}$ is transformed as

$$
\sigma_{s}^{0}=P_{s} P^{\prime-\frac{3}{2}} \bar{P}^{\prime \frac{1}{2}} \sigma^{\prime 0}-\partial_{\bar{z}}\left(P_{s} \sqrt{P^{\prime} \bar{P}^{\prime}} \partial_{\bar{z}} u^{\prime}\right)+\sqrt{P^{\prime} \bar{P}^{\prime}} \partial_{\bar{z}} u^{\prime} \partial_{u}\left(\sqrt{P^{\prime} \bar{P}^{\prime}} \partial_{\bar{z}} u^{\prime}\right) .
$$

$P^{\prime}, \bar{P}^{\prime}$ and $\sigma^{\prime 0}$ are scalar fields, hence

$$
P^{\prime}\left(u^{\prime}, z^{\prime}, \bar{z}^{\prime}\right)=P(u, z, \bar{z}), \bar{P}^{\prime}\left(u^{\prime}, z^{\prime}, \bar{z}^{\prime}\right)=\bar{P}(u, z, \bar{z}), \quad \sigma^{\prime 0}\left(u^{\prime}, z^{\prime}, \bar{z}^{\prime}\right)=\sigma^{0}(u, z, \bar{z}) .
$$

We can just drop the prime

$$
\sigma_{s}^{0}=P_{s} P^{-\frac{3}{2}} \bar{P}^{\frac{1}{2}} \sigma^{0}-\partial_{\bar{z}}\left(P_{s} \sqrt{P \bar{P}} \partial_{\bar{z}} u^{\prime}\right)+\sqrt{P \bar{P}} \partial_{\bar{z}} u^{\prime} \partial_{u}\left(\sqrt{P \bar{P}} \partial_{\bar{z}} u^{\prime}\right) .
$$

Since we have put the solution in $(u, z, \bar{z})$ coordinates with $S^{2} \times \mathbb{R}$ boundary topology, the standard displacement memory formula works. It is just the change of $\sigma_{s}^{0}$ at early time $u_{i}$ and late time $u_{f}$ in this coordinates.

Alternatively, in $\left(u^{\prime}, z^{\prime}, \bar{z}^{\prime}\right)$ coordinates, one can define

$$
{\sigma_{s}^{\prime}}_{s}^{0}=P_{s} P^{\prime}\left(u^{\prime}, z^{\prime}, \bar{z}^{\prime}\right)^{-\frac{3}{2}} \bar{P}^{\prime}\left(u^{\prime}, z^{\prime}, \bar{z}^{\prime}\right)^{\frac{1}{2}} \sigma^{\prime 0}\left(u^{\prime}, z^{\prime}, \bar{z}^{\prime}\right)+P_{s} \partial_{\bar{z}^{\prime}}^{2}\left[\int_{0}^{u^{\prime}} d v \sqrt{P^{\prime} \bar{P}^{\prime}}\right] .
$$

This is the Weyl invariant part of $\sigma^{\prime 0}\left(u^{\prime}, z^{\prime}, \bar{z}^{\prime}\right)$ [41], namely it is unchanged as a function of their variables under Weyl transformation. This $\sigma_{s}^{\prime 0}$ determines the memory effect in

\footnotetext{
${ }^{2}$ One should not confuse this with the notation in appendix A where $(u, z, \bar{z})$ is used for a general solution with arbitrary 2 surface boundary.

${ }^{3}$ The full coordinate transformation is given in the form of an asymptotic expansion, but only the leading terms are involved in deriving the transformation law of jthe relevant fields.
} 
$\left(u^{\prime}, z^{\prime}, \bar{z}^{\prime}\right)$ coordinates. The displacement memory can be derived from the change of $\sigma_{s}^{\prime 0}$ at early time $u_{i}^{\prime}$ and late time $u_{f}^{\prime}$ and is given by

$$
\begin{aligned}
\left.\Delta \sigma_{s}^{\prime 0}\right|_{u_{i}^{\prime}} ^{u_{f}^{\prime}}= & P_{s} P^{\prime}\left(u_{f}^{\prime}, z^{\prime}, \bar{z}^{\prime}\right)^{-\frac{3}{2}} \bar{P}^{\prime}\left(u_{f}^{\prime}, z^{\prime}, \bar{z}^{\prime}\right)^{\frac{1}{2}} \sigma^{\prime 0}\left(u_{f}^{\prime}, z^{\prime}, \bar{z}^{\prime}\right) \\
& -P_{s} P^{\prime}\left(u_{i}^{\prime}, z^{\prime}, \bar{z}^{\prime}\right)^{-\frac{3}{2}} \bar{P}^{\prime}\left(u_{i}^{\prime}, z^{\prime}, \bar{z}^{\prime}\right)^{\frac{1}{2}} \sigma^{\prime 0}\left(u_{i}^{\prime}, z^{\prime}, \bar{z}^{\prime}\right)+P_{s} \partial_{\bar{z}^{\prime}}^{2}\left[\int_{u_{i}^{\prime}}^{u_{f}^{\prime}} d v \sqrt{P^{\prime} \bar{P}^{\prime}}\right]
\end{aligned}
$$

We would like to comment more, from the geometric point of view, on the case of an arbitrary 2 surface. From the geodesic equation

$$
\nabla_{n} n=-(\gamma+\bar{\gamma}) n_{\mu}+\bar{\nu} \bar{m}_{\mu}+\nu m_{\mu}
$$

we find that $n$ will not be tangent to a null geodesic on null infinity when $P$ is $u$-dependent. $\mu^{0}$ and $\lambda^{0}$ are the asymptotic expansion and shear of $n$ respectively [39]. In the unit 2 sphere case, $n$ is tangent to null geodesics with affine parameter $u$. The Weyl tensor $\Psi_{s 3}^{0}$ and $\Psi_{s 4}^{0}$ are completely determined by the asymptotic shear of the null geodesic congruence. We call them the news functions as they indicate the existence of gravitational waves. However, in the case of arbitrary 2 surface, the asymptotic shear $\lambda^{0}$ will not only be controlled by gravitational waves, but will be also affected by the reference system. Nevertheless, we can define

$$
\lambda_{s}^{0}=P_{s}^{2}\left(\frac{\lambda^{0}}{\bar{P}^{2}}+\frac{\partial_{z}^{2} \sqrt{P \bar{P}}}{\sqrt{P \bar{P}}}\right),
$$

so that $\lambda_{s}^{0}$ measures only the gravitational wave contribution, while the second piece in the parentheses on the right hand side is purely the reference system effect.

The special choice of boundary topology in the standard treatment of the memory effect represents physical space-times which contain isolated systems, with no geometrical or topological information from outside world [42]. An arbitrary 2 surface boundary is certainly compatible with the condition that no geometrical information is coming from the outside world. However, topological information can not be avoided, for instance a null geodesic on null infinity may not be complete. Hence the Weyl transformations are usually singular in such case. Another interesting transformation that involves singularities are the so-called super-rotations [43-45]. The physical status of finite super-rotations and the transition between such states is related to the breaking of a cosmic string via quantum black hole pair nucleation [46]. It is definitely of interest to investigate the physical consequence of super-rotations in memory effects elsewhere.

\section{Robinson-Trautman waves}

In this short section, we are ready to clarify the puzzle about the memory effect of the Robinson-Trautman waves via the generalized displacement memory formula developed in the previous section. The Robinson-Trautman metric was originally derived in [25] to demonstrate a very simple kind of spherical radiation. Adapted to our notation, the 
metric is ${ }^{4}$

$$
d s^{2}=2\left(-r \partial_{u} \ln P+P^{2} \partial_{z} \partial_{\bar{z}} \ln P+\frac{\Psi_{2}^{0}}{r}\right) d u^{2}+2 d u d r-2 \frac{r^{2}}{P^{2}} d z d \bar{z}
$$

where $\Psi_{2}^{0}$ is a real constant and $P$ is a real arbitrary function of $(u, z, \bar{z})$ satisfying

$$
3 \Psi_{2}^{0} \partial_{u} P+P^{3} \partial_{\bar{z}}^{2} P \partial_{z}^{2} P-P^{4} \partial_{z}^{2} \partial_{\bar{z}}^{2} P=0 .
$$

In the NP formalism, the solution is given by

$$
\begin{aligned}
& \Psi_{0}=\Psi_{1}=\sigma=\lambda=\tau=X^{A}=\omega=0, \\
& \Psi_{2}=\frac{\Psi_{2}^{0}}{r^{3}}, \\
& \mu_{0}=-P^{2} \partial \bar{\partial} \ln P \\
& \Psi_{3}=\frac{P \partial_{z} \mu^{0}}{r^{2}}, \\
& \Psi_{4}=\frac{-\partial_{z}\left(P^{2} \partial_{z} \partial_{u} \ln P\right)}{r}-\frac{P^{2} \partial_{z} \partial_{\bar{z}} \mu^{0}}{r^{2}}, \\
& \rho=-\frac{1}{r} \\
& \alpha=\frac{\partial_{z} P}{2 r}, \\
& \beta=-\frac{\partial_{\bar{z}} P}{2 r}, \quad \mu=\frac{\mu_{0}}{r}-\frac{\Psi_{2}^{0}}{r^{2}}, \\
& \gamma=-\frac{1}{2} \partial_{u} \ln P-\frac{\Psi_{2}^{0}}{r^{2}} \\
& \nu=-P \partial_{z} \partial_{u} \ln P-\frac{P \partial_{z} \mu^{0}}{r}, \\
& U=r \partial_{u} \ln P+\mu^{0}-\frac{\Psi_{2}^{0}}{r}, \\
& L^{z}=0, \\
& L^{\bar{z}}=\frac{P}{r},
\end{aligned}
$$

where $\Psi_{2}^{0}$ is a real constant. As explained in the previous section, $n$ is not tangent to a null geodesic on null infinity in this case. Both gravitational radiation and the effect of the reference system will contribute to the asymptotic shear of the null congruence $\lambda^{0}$ that $n$ is tangent to. Their contributions happen to cancel, namely $\lambda^{0}=0$. The standard displacement memory formula does not apply in this situation. To eliminate the reference effect, one needs to use a "good" reference system with time coordinate $\tilde{u}=\int_{0}^{u} d v \frac{P}{P_{s}}$. However, according to (2.6), the displacement memory effect from gravitational radiation can be obtained directly in the original coordinates. It is just

$$
P_{s} \partial_{\bar{z}}^{2}\left(\int_{u_{i}}^{u_{f}} d v P\right)
$$

To the best of our knowledge, non-trivial explicit solutions of equation (3.2) rarely exist. One may need to turn to numerical methods to find the exact value of displacement memory.

\section{Spin memory effect}

Recently, Pasterski, Strominger, and Zhiboedov discovered a new type of gravitational memory, the so-called spin memory effect [22]. They proposed that the observational effect of spin memory is the relative time delay of different light rays at very large radial distance $r_{0}$. In this section, we will provide a new observational effect by looking at time-like geodesics with affine parameter very close to null infinity.

\footnotetext{
${ }^{4}$ To compare with the solution in [47], $P$ should be multiplied by a factor of $\frac{1}{2}$.
} 
The observer will be constrained to a fixed radial distance $r_{0}$ which is very far from the gravitational source, e.g. on the earth. The $r=r_{0}$ hypersurface is time-like, its induced metric can be derived easily from the most general NU solutions in appendix A. Up to relevant orders, the induced metric is given by

$$
\begin{aligned}
d s^{2}= & {\left[1+\frac{\Psi_{2}^{0}+\bar{\Psi}_{2}^{0}}{r}-\frac{\bar{\partial} \Psi_{1}^{0}+ð \bar{\Psi}_{1}^{0}}{r^{2}}+O\left(r^{-3}\right)\right] d u^{2} } \\
& -2\left[\frac{\partial \bar{\sigma}^{0}}{P_{s}}-\frac{2 \bar{\Psi}_{1}^{0}}{3 P_{s} r}+O\left(r^{-2}\right)\right] d u d z-2\left[\frac{\bar{\partial} \sigma^{0}}{P_{s}}-\frac{2 \Psi_{1}^{0}}{3 P_{s} r}+O\left(r^{-2}\right)\right] d u d \bar{z} \\
& -\left[2 \frac{\bar{\sigma}^{0} r}{P_{s}^{2}}-\frac{\bar{\Psi}_{0}^{0}}{3 P_{s} r}+O\left(r^{-2}\right)\right] d z^{2}-\left[2 \frac{\sigma^{0} r}{P_{s}^{2}}-\frac{\Psi_{0}^{0}}{3 P_{s} r}+O\left(r^{-2}\right)\right] d \bar{z}^{2} \\
& -2\left[\frac{r^{2}}{P_{s}^{2}}+\frac{\sigma^{0} \bar{\sigma}^{0}}{P_{s}^{2}}+O\left(r^{-2}\right)\right] d z d \bar{z} .
\end{aligned}
$$

We will work in the unit 2 sphere case. An arbitrary 2 surface can be mapped onto the unit 2 sphere by a Weyl transformation as discussed in previous section (see more details in [41]).

Free falling observers on this hypersurface will travel along time-like geodesics. Supposing that the vector $V$ is tangent to a time-like geodesic, it should satisfy the geodesic equation

$$
\bar{\nabla}_{V} V=0
$$

where $\bar{\nabla}$ is the covariant derivative on this 3 dimensional hypersurface. Actually, $V$ is induced from a 4 dimensional vector $\tilde{V}$. When $r \rightarrow \infty$, a 4 dimensional time-like vector $\tilde{V}$ will either vanish or be proportional to the null basis $n$ (in the Newman-Penrose formalism) which is the generator of null infinity, because the light-cone will be squashed to a line on null infinity. Hence $V$ should have the following asymptotic behavior:

$$
V^{u}=1+\sum_{a=1}^{\infty} \frac{V_{a}^{u}}{r^{a}}, \quad V^{z}=\sum_{a=2}^{\infty} \frac{V_{a}^{z}}{r^{a}} .
$$

Then we need to solve the geodesic equation order by order. The solution is (up to relevant order):

$$
\begin{aligned}
V_{1}^{u}= & -\frac{\Psi_{2}^{0}+\bar{\Psi}_{2}^{0}}{2}+V_{1 I}^{u}(z, \bar{z}) \\
V_{2}^{u}= & \frac{1}{6}\left(\bar{\partial} \Psi_{1}^{0}+\partial \bar{\Psi}_{1}^{0}\right)-\partial \bar{\sigma}^{0} \bar{\partial} \sigma^{0}+\frac{3}{8}\left(\Psi_{2}^{0}+\bar{\Psi}_{2}^{0}\right)^{2}-\frac{1}{2} V_{1 I}^{u}\left(\Psi_{2}^{0}+\bar{\Psi}_{2}^{0}\right)+V_{2 I}^{u}(z, \bar{z}) \\
V_{2}^{z}= & -P_{s} \bar{\partial} \sigma^{0}+V_{2 I}^{z}(z, \bar{z}), \\
V_{3}^{z}= & P_{s}\left[2 \widetilde{\partial} \bar{\sigma}^{0} \sigma^{0}+\frac{2}{3} \Psi_{1}^{0}+\frac{1}{2} \bar{\partial} \sigma^{0}\left(\Psi_{2}^{0}+\bar{\Psi}_{2}^{0}\right)\right]-P_{s} \int d v \frac{\partial\left(\Psi_{2}^{0}+\bar{\Psi}_{2}^{0}\right)}{2} \\
& -P_{s} \bar{\partial} \sigma^{0} V_{1 I}^{u}-2 \sigma^{0} V_{2 I}^{\bar{z}}+V_{3 I}^{z}(z, \bar{z}),
\end{aligned}
$$

where $V_{1 I}^{u}, \quad V_{2 I}^{u}, \quad V_{2 I}^{z}, \quad V_{3 I}^{z}$ are integration constants that indicate the initial velocity of the observer. We will now set all of them to be zero as we require the observer is static 
initially. At $r_{0}^{-2}$ order, $V$ has angular components due to the presence of gravitational waves characterized by $\sigma^{0}$. In other words, gravitational radiation forces free falling timelike particles to rotate. Since $V$ is time-like, the infinitesimal change of the proper time of the observer can be derived from the co-vector. It is just:

$$
\begin{aligned}
d \chi & =d u+\frac{1}{2 r_{0}}\left(\left(\Psi_{2}^{0}+\bar{\Psi}_{2}^{0}\right) d u+\frac{\int \bar{\partial}\left(\Psi_{2}^{0}+\bar{\Psi}_{2}^{0}\right) d v}{P_{s}} d z+\frac{\int ð\left(\Psi_{2}^{0}+\bar{\Psi}_{2}^{0}\right) d v}{P_{s}} d \bar{z}\right)+O\left(r_{0}^{-2}\right) \\
& =d\left(u+\frac{1}{2 r_{0}} \int\left(\Psi_{2}^{0}+\bar{\Psi}_{2}^{0}\right) d v\right)+O\left(r_{0}^{-2}\right)
\end{aligned}
$$

where $\chi$ is the proper time. Defining

$$
\mathcal{M}=\frac{1}{2} \int\left(\Psi_{2}^{0}+\bar{\Psi}_{2}^{0}\right) d v
$$

between two space-time points $\left(u_{i}, z_{i}, \bar{z}_{i}\right)$ and $\left(u_{f}, z_{f}, \bar{z}_{f}\right)$ on this geodesic, the change in proper time is

$$
\Delta \chi=\Delta u+\frac{1}{r_{0}} \Delta \mathcal{M}+O\left(r_{0}^{-2}\right) .
$$

Clearly, the observer receives a time delay at order $\frac{1}{r}$. We want to emphasize that $\Delta \mathcal{M}$ is angle dependent. Thus, both the Bondi mass aspect of a massive object and gravitational radiation contribute to the time delay. $\Delta \mathcal{M}$ is constrained by the time evolution equation

$$
\partial_{u} \Psi_{1}^{0}=ð \Psi_{2}^{0}-2 \sigma^{0} \partial_{u} \partial \bar{\sigma}^{0}
$$

and it is completely fixed by the change of the angular momentum aspect as ${ }^{5}$

$$
\bar{\partial} \precsim \Delta \mathcal{M}=\Delta\left(\frac{1}{2} \bar{\partial} \Psi_{1}^{0}+\frac{1}{2} ð \bar{\Psi}_{1}^{0}+\bar{\partial} \sigma^{0} \precsim \bar{\sigma}^{0}\right)+\int\left(\sigma^{0} \bar{\partial} \dot{\sigma}^{0}+\bar{\sigma}^{0} \precsim \bar{\partial} \dot{\sigma}^{0}\right) d v,
$$

up to a real constant. This is very similar to the displacement memory $[2,7]$ in the sense that it includes the linear piece

$$
\Delta\left(\frac{1}{2} \bar{\partial} \Psi_{1}^{0}+\frac{1}{2} \partial \bar{\Psi}_{1}^{0}+\bar{\partial} \sigma^{0} \partial \bar{\sigma}^{0}\right)
$$

and the non-linear piece

$$
\int\left(\sigma^{0} \bar{\varnothing} \dot{\sigma}^{0}+\bar{\sigma}^{0} \partial \bar{\varnothing} \dot{\sigma}^{0}\right) d v
$$

The effect of the integration constant in (4.8) can be eliminated by choosing a ring of freely falling observers who are initially static and synchronized. ${ }^{6}$ The difference in the

\footnotetext{
${ }^{5}$ See also [22] for the relation of the angular momentum flux to the spin memory.

${ }^{6} \mathrm{It}$ is meaningful to point out that displacement memory and spin memory happen at the same time when $\Delta \mathcal{M}$ is angle dependent. There is only displacement memory no spin memory when $\Delta \mathcal{M}$ has no angular dependence. In such case, the changes in proper time of a ring of freely falling observers are the same. In this sense, we say displacement memory does not contribute to a relative time delay for a ring of free falling observers (see also appendix A of [12]).
} 
proper time of every observer on this ring at a later time can memorize the waveform of the gravitational waves. This is another observational effect of the spin memory.

Since we have $V=n$ when $r \rightarrow \infty$, the leading piece of the shear of the time-like geodesic congruence is just $\frac{\lambda^{0}}{r}$. The ring of free falling observers will observe two memory effects: the displacement memory that will squash and stretch the ring and the time delay that will cause the difference of the proper time of nearby observers.

\section{Conjugate points}

To measure the time delay effect, one may expect an even simpler ideal experiment if there are conjugate points on the time-like geodesics, which is possible in curved space-time. Two free falling observers are launched with certain initial velocities at the same point. Then they will meet again at the conjugate point where they can compare their proper time. However, such an experiment is extremely hard to arrange. Because conjugate points on a time-like geodesic are very very far from each other in the asymptotic region in our set-up, though a time-like geodesic does have conjugate points [48] in such cases. In order to show that, we will consider part of a time-like geodesic, namely the length of this part of the geodesic is much smaller than $r_{0}$. Then the $\frac{1}{r}$ expansion can be applied. In the end, we will prove that there are no conjugate points on this part of the geodesic.

A solution $T^{c}$ of the geodesic deviation equation

$$
\bar{\nabla}_{V}\left(\bar{\nabla}_{V} T^{c}\right)=-R_{a b d}^{c} T^{b} V^{a} V^{d}
$$

is called a Jacobi field on the geodesic that $V$ is tangent to. Two points $p$ and $q$ are conjugate points along the geodesic if there exists a non-zero Jacobi field $T^{c}$ along the geodesic that vanishes at $p$ and $q[48]$. According to the induced metric (4.1), $R_{a b d}{ }^{c} V^{a} V^{d}=O\left(r^{-1}\right)$, so the leading piece of the Geodesic deviation equation is

$$
\partial_{u}^{2} T_{0}^{a}(u, z, \bar{z})=0
$$

where $T_{0}^{a}(u, z, \bar{z})$ is the leading term of $T^{a}$ in the $\frac{1}{r}$ expansion. Then $T_{0}^{a}$ is given by

$$
T_{0}^{a}=u T_{01}^{a}(z, \bar{z})+T_{02}^{a}(z, \bar{z}) .
$$

Naively, one can find an infinite number of possible choices of $T_{0}^{a}$ that allow two points $\left(u_{i}, z_{i}, \bar{z}_{i}\right)$ and $\left(u_{f}, z_{f}, \bar{z}_{f}\right)$ from the geodesic to be conjugate points, namely

$$
\begin{aligned}
u_{i} T_{01}^{a}\left(z_{i}, \bar{z}_{i}\right)+T_{02}^{a}\left(z_{i}, \bar{z}_{i}\right) & =0, \\
u_{f} T_{01}^{a}\left(z_{f}, \bar{z}_{f}\right)+T_{02}^{a}\left(z_{f}, \bar{z}_{f}\right) & =0 .
\end{aligned}
$$

However, the change of the angular coordinates on this geodesic is very tiny and proportional to $\frac{1}{r}$. Then the second equation $u_{f} T_{01}^{a}\left(z_{f}, \bar{z}_{f}\right)+T_{02}^{a}\left(z_{f}, \bar{z}_{f}\right)=0$ becomes

$$
u_{f} T_{01}^{a}\left(z_{i}, \bar{z}_{i}\right)+T_{02}^{a}\left(z_{i}, \bar{z}_{i}\right)+O\left(r^{-1}\right)=0 .
$$


Hence the condition of having $\left(u_{i}, z_{i}, \bar{z}_{i}\right)$ and $\left(u_{f}, z_{f}, \bar{z}_{f}\right)$ be conjugate points is reduced to

$$
\begin{aligned}
u_{i} T_{01}^{a}\left(z_{i}, \bar{z}_{i}\right)+T_{02}^{a}\left(z_{i}, \bar{z}_{i}\right) & =0, \\
u_{f} T_{01}^{a}\left(z_{i}, \bar{z}_{i}\right)+T_{02}^{a}\left(z_{i}, \bar{z}_{i}\right) & =0,
\end{aligned}
$$

but $u_{i} \neq u_{f}$. Obviously, there is no such solution.

Thus we can never find two points that are not very far from each other on one geodesic where $T^{a}=0$ at leading order. Conceptually, this is expected since geodesics in flat (Minkowski) space-times do not have conjugate points. Now we have just shown that conjugate points are not "close" to each other in the asymptotic region in asymptotically flat space-times though they do exist.

\section{Discussions}

In this work, we first derived the formula for the displacement memory effect for the case with an arbitrary 2 surface boundary topology. Via a Weyl transformation, it can be mapped into the unit 2 sphere. Then the standard formula for the displacement memory applies. This leads us to a direct derivation of the displacement memory formula in the original form of the solutions. Secondly, we proposed a new observational effect of the spin memory. It is a time delay of time-like free falling observers.

The discovery of spin memory was originally inspired by the connection between the gravitational memory effect and Weinberg's soft graviton theorem. The displacement memory and spin memory correspond to the leading and sub-leading soft graviton theorem, respectively. The novel results in [21] show that soft graviton theorems exist even at third order in the low-energy expansion. There are indeed some positive signs indicating a third gravitational memory effect. On the one hand, the two known memory formulas are completely controlled by the time evolution equations of the Weyl tensors:

$$
\partial_{u} \Psi_{2}^{0}=-\partial_{u} \check{\partial}^{2} \bar{\sigma}^{0}-\sigma^{0} \partial_{u}^{2} \bar{\sigma}^{0},
$$

and

$$
\partial_{u} \Psi_{1}^{0}=\partial \Psi_{2}^{0}-2 \sigma^{0} \partial_{u} \partial \bar{\sigma}^{0}
$$

in unit 2 sphere case. There is indeed a third time evolution equation

$$
\partial_{u} \Psi_{0}^{0}=\partial \Psi_{1}^{0}+3 \sigma^{0} \Psi_{2}^{0} .
$$

The asymptotic shear $\sigma^{0}$ is constrained by the imaginary part of this equation through some tedious but not difficult calculations (it is more clear in the linearized gravity case [49]). On the other hand, displacement and spin memories are related to the energy flux and the angular momentum flux through null infinity. Newman and Penrose $[50,51]$ discovered more gravitationally-conserved quantities that may account for the possible third gravitational memory (see also recent relevant developments [52-55]).

We have shown the power of Weyl transformations with a precise example in this work. Actually, the action of the full $\mathrm{BMS}_{4}$ group combined with Weyl transformations on the Newman-Unti solution space was given in [41]. It would be very meaningful to compute the transformation law of the $\mathrm{BMS}_{4}$ current, especially the action of a Weyl transformation elsewhere [56]. 


\section{Acknowledgments}

The authors thank Glenn Barnich and Jun-Bao Wu for useful discussions and additionally Jun-Bao Wu again for critical comments on our computations. This work is supported in part by the China Postdoctoral Science Foundation (Grant No. 2017M620908), by the National Natural Science Foundation of China (Grant Nos. 11575286, 11731001, 11475179, and 11575202).

\section{A Newman-Unti solution in the Newman-Penrose formalism}

The Newman-Penrose formalism [39] is a tetrad formalism with a special choice of four null basis vectors $e_{1}=l, e_{2}=n, e_{3}=m, e_{4}=\bar{m}$, where $l$ and $n$ are real while $m$ and $\bar{m}$ are complex conjugates of each other. The null basis vectors satisfy orthogonality conditions $l \cdot m=l \cdot \bar{m}=n \cdot m=n \cdot \bar{m}=0$ and normalization conditions $l \cdot n=1, m \cdot \bar{m}=-1$. The connection coefficients are called spin coefficients in the NP formalism with special Greek symbols (we will follow the convention of [57]). The metric is constructed from

$$
g_{\mu \nu}=n_{\mu} l_{\nu}+l_{\mu} n_{\nu}-m_{\mu} \bar{m}_{\nu}-m_{\nu} \bar{m}_{\mu}
$$

In the NP formalism, it is always possible to impose

$$
\pi=\kappa=\epsilon=0, \rho=\bar{\rho}, \tau=\bar{\alpha}+\beta,
$$

which means that $l$ is tangent to a null geodesic with affine parameter and the congruence of the null geodesic is hypersurface orthogonal, i.e. $l$ will be proportional to the gradient of a scalar field. It is convenient to take this scalar field as coordinate $u=x^{1}$ and set the affine parameter as coordinate $r=x^{2}$. To satisfy the orthogonality and normalization conditions, the basis vectors and the cotetrad must have the form

$$
\begin{aligned}
& n=\frac{\partial}{\partial u}+U \frac{\partial}{\partial r}+X^{A} \frac{\partial}{\partial x^{A}}, \quad l=\frac{\partial}{\partial r}, \quad m=\omega \frac{\partial}{\partial r}+L^{A} \frac{\partial}{\partial x^{A}}, \\
& n=\left[-U-X^{A}\left(\bar{\omega} L_{A}+\omega \bar{L}_{A}\right)\right] d u+d r+\left(\omega \bar{L}_{A}+\bar{\omega} L_{A}\right) d x^{A}, \\
& l=d u, \quad m=-X^{A} L_{A} d u+L_{A} d x^{A},
\end{aligned}
$$

where $L_{A} L^{A}=0, L_{A} \bar{L}^{A}=-1$. The main condition of approaching flatness at infinity is $\Psi_{0}=\frac{\Psi_{0}^{0}}{r^{5}}+O\left(r^{-6}\right)$. Newman and Unti [40] derived the most general solutions of the NP system that preserve the conditions listed above. The asymptotic expansion of all components in stereographic coordinates $(z, \bar{z})$ is given by:

$$
\begin{aligned}
\Psi_{0} & =\frac{\Psi_{0}^{0}(u, z, \bar{z})}{r^{5}}+O\left(r^{-6}\right), \\
\Psi_{1} & =\frac{\Psi_{1}^{0}(u, z, \bar{z})}{r^{4}}-\frac{\bar{\partial} \Psi_{0}^{0}}{r^{5}}+O\left(r^{-6}\right), \\
\Psi_{2} & =\frac{\Psi_{2}^{0}(u, z, \bar{z})}{r^{3}}-\frac{\bar{\partial} \Psi_{1}^{0}}{r^{4}}+O\left(r^{-5}\right), \\
\Psi_{3} & =\frac{\Psi_{3}^{0}}{r^{2}}-\frac{\overline{\bar{\partial}} \Psi_{2}^{0}}{r^{3}}+O\left(r^{-4}\right),
\end{aligned}
$$




$$
\begin{aligned}
& \Psi_{4}=\frac{\Psi_{4}^{0}}{r}-\frac{\bar{\gamma} \Psi_{3}^{0}}{r^{2}}+O\left(r^{-3}\right), \\
& \rho=-\frac{1}{r}-\frac{\sigma^{0} \bar{\sigma}^{0}}{r^{3}}+O\left(r^{-5}\right), \\
& \tau=-\frac{\Psi_{1}^{0}}{r^{3}}+O\left(r^{-4}\right) \\
& \sigma=\frac{\sigma^{0}(u, z, \bar{z})}{r^{2}}+\left(\sigma^{0} \sigma^{0} \bar{\sigma}^{0}-\frac{1}{2} \Psi_{0}^{0}\right) r^{-4}+O\left(r^{-5}\right), \\
& \alpha=\frac{\alpha^{0}}{r}+\frac{\bar{\sigma}^{0} \bar{\alpha}^{0}}{r^{2}}+\frac{\sigma^{0} \bar{\sigma}^{0} \alpha^{0}}{r^{3}}+O\left(r^{-4}\right), \\
& \beta=-\frac{\bar{\alpha}^{0}}{r}-\frac{\sigma^{0} \alpha^{0}}{r^{2}}-\frac{\sigma^{0} \bar{\sigma}^{0} \bar{\alpha}^{0}+\frac{1}{2} \Psi_{1}^{0}}{r^{3}}+O\left(r^{-4}\right), \\
& \mu=\frac{\mu^{0}}{r}-\frac{\sigma^{0} \lambda^{0}+\Psi_{2}^{0}}{r^{2}}+\left(\sigma^{0} \bar{\sigma}^{0} \mu^{0}+\frac{1}{2} \bar{\partial} \Psi_{1}^{0}\right) r^{-3}+O\left(r^{-4}\right), \\
& \lambda=\frac{\lambda^{0}}{r}-\frac{\bar{\sigma}^{0} \mu^{0}}{r^{2}}+\left(\sigma^{0} \bar{\sigma}^{0} \lambda^{0}+\frac{1}{2} \bar{\sigma}^{0} \Psi_{2}^{0}\right) r^{-3}+O\left(r^{-4}\right), \\
& \gamma=\gamma^{0}-\frac{\Psi_{2}^{0}}{r^{2}}+\frac{1}{6}\left(2 \bar{\varnothing} \Psi_{1}^{0}+\alpha^{0} \Psi_{1}^{0}-\bar{\alpha}^{0} \bar{\Psi}_{1}^{0}\right) r^{-3}+O\left(r^{-4}\right), \\
& \nu=\nu^{0}-\frac{\Psi_{3}^{0}}{r}+\frac{\overline{\bar{\partial}} \Psi_{2}^{0}}{2 r^{2}}+O\left(r^{-3}\right), \\
& X^{z}=\frac{\bar{P} \Psi_{1}^{0}}{6 r^{3}}+O\left(r^{-4}\right) \text {, } \\
& \omega=\frac{\bar{\partial} \sigma^{0}}{r}-\frac{\sigma^{0} \precsim \bar{\sigma}^{0}+\frac{1}{2} \Psi_{1}^{0}}{r^{2}}+O\left(r^{-3}\right), \\
& U=-r\left(\gamma^{0}+\bar{\gamma}^{0}\right)+\mu^{0}-\frac{\Psi_{2}^{0}+\bar{\Psi}_{2}^{0}}{2 r}+\frac{1}{6}\left(\bar{\delta} \Psi_{1}^{0}+ð \bar{\Psi}_{1}^{0}\right) r^{-2}+O\left(r^{-3}\right), \\
& L^{z}=-\frac{\sigma^{0} \bar{P}(u, z, \bar{z})}{r^{2}}-\frac{\bar{P}}{r^{4}}\left(\sigma^{0^{2}} \bar{\sigma}^{0}-\frac{1}{6} \Psi_{0}^{0}\right)+O\left(r^{-5}\right), \\
& L^{\bar{z}}=\frac{P(u, z, \bar{z})}{r}+\frac{\sigma^{0} \bar{\sigma}^{0} P}{r^{3}}+O\left(r^{-5}\right), \\
& L_{z}=-\frac{r}{\bar{P}}+O\left(r^{-3}\right) \text {, } \\
& L_{\bar{z}}=-\frac{\sigma^{0}}{P}+\frac{\Psi_{0}^{0}}{6 P r^{2}}+O\left(r^{-3}\right) \text {, }
\end{aligned}
$$

where

$$
\begin{aligned}
& \alpha^{0}=\frac{1}{2} \bar{P} \partial_{z} \ln P, \quad \mu^{0}=-\frac{1}{2} P \bar{P} \partial_{z} \partial_{\bar{z}} \ln P \bar{P}, \\
& \lambda^{0}=\partial_{u} \bar{\sigma}^{0}+\bar{\sigma}^{0}\left(3 \gamma^{0}-\bar{\gamma}^{0}\right), \\
& \gamma^{0}=-\frac{1}{2} \partial_{u} \ln \bar{P}, \quad \nu^{0}=\overline{\mathrm{\partial}}\left(\gamma^{0}+\bar{\gamma}^{0}\right), \\
& \Psi_{2}^{0}-\bar{\Psi}_{2}^{0}=\bar{\partial}^{2} \sigma^{0}-\partial^{2} \bar{\sigma}^{0}+\bar{\sigma}^{0} \bar{\lambda}^{0}-\sigma^{0} \lambda^{0}, \\
& \Psi_{3}^{0}=\bar{\partial} \mu^{0}-\bar{\partial} \lambda^{0}, \quad \Psi_{4}^{0}=\bar{\partial} \nu^{0}-\partial_{u} \lambda^{0}-4 \gamma^{0} \lambda^{0},
\end{aligned}
$$




\begin{tabular}{|c|c|c|c|c|c|c|c|c|c|c|c|c|}
\hline & $\partial$ & $\partial_{u}$ & $\gamma^{0}$ & $\nu^{0}$ & $\mu^{0}$ & $\sigma^{0}$ & $\lambda^{0}$ & $\Psi_{4}^{0}$ & $\Psi_{3}^{0}$ & $\Psi_{2}^{0}$ & $\Psi_{1}^{0}$ & $\Psi_{0}^{0}$ \\
\hline $\mathrm{s}$ & 1 & 0 & 0 & -1 & 0 & 2 & -2 & -2 & -1 & 0 & 1 & 2 \\
\hline
\end{tabular}

Table 1. Spin weights.

$$
\begin{aligned}
\partial_{u} \Psi_{0}^{0}+\left(\gamma^{0}+5 \bar{\gamma}^{0}\right) \Psi_{0}^{0} & =ð \Psi_{1}^{0}+3 \sigma^{0} \Psi_{2}^{0}, \\
\partial_{u} \Psi_{1}^{0}+2\left(\gamma^{0}+2 \bar{\gamma}^{0}\right) \Psi_{1}^{0} & =\partial \Psi_{2}^{0}+2 \sigma^{0} \Psi_{3}^{0}, \\
\partial_{u} \Psi_{2}^{0}+3\left(\gamma^{0}+\bar{\gamma}^{0}\right) \Psi_{2}^{0} & =\partial \Psi_{3}^{0}+\sigma^{0} \Psi_{4}^{0}, \\
\partial_{u} \Psi_{3}^{0}+2\left(2 \gamma^{0}+\bar{\gamma}^{0}\right) \Psi_{3}^{0} & =\partial \Psi_{4}^{0} .
\end{aligned}
$$

The "क" operator is defined by

$$
\begin{aligned}
& ð \eta^{s}=P \bar{P}^{-s} \partial_{\bar{z}}\left(\bar{P}^{s} \eta^{s}\right)=P \partial_{\bar{z}} \eta^{s}+2 s \bar{\alpha}^{0} \eta^{s}, \\
& \bar{\jmath} \eta^{s}=\bar{P} P^{s} \partial_{z}\left(P^{-s} \eta^{s}\right)=\bar{P} \partial_{z} \eta^{s}-2 s \alpha^{0} \eta^{s},
\end{aligned}
$$

where $s$ is the spin weight of the field $\eta$. The spin weights of relevant fields are listed below in table 1 .

\section{B Weyl transformation of solutions}

In the Newman-Penrose formalism, a gauge transformation is a combination of a change of coordinates and a local Lorentz transformation which is described in the standard three classes of rotations [57]. The residual gauge transformations preserving the gauge condition and asymptotic behaviors of Newman-Unti solutions were derived by Barnich and Troessaert [41] recently. A pure Weyl transformation is characterized by

$$
z^{\prime}=z, \quad u^{\prime}=\int_{0}^{u} d v e^{E_{R}}, \quad P^{\prime}=P e^{-E_{R}} .
$$

The transformation law of the data that characterizes the asymptotic solution is given by

$$
\begin{aligned}
& \sigma^{\prime 0}=e^{-E_{R}}\left[\sigma^{0}+ð\left(e^{-E_{R}} \partial u^{\prime}\right)-\left(e^{-E_{R}} \partial u^{\prime}\right)\left(\partial_{u}+\bar{\gamma}^{0}-\gamma^{0}\right)\left(e^{-E_{R}} \partial u^{\prime}\right)\right],
\end{aligned}
$$

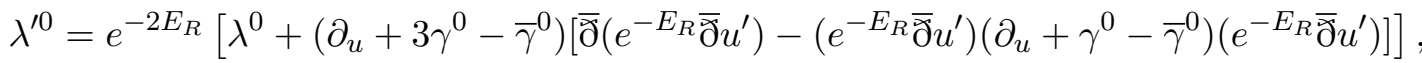

$$
\begin{aligned}
& \Psi_{4}^{\prime 0}=e^{-3 E_{R}}\left[\Psi_{4}^{0}\right] \text {, } \\
& \Psi_{3}^{\prime 0}=e^{-3 E_{R}}\left[\Psi_{3}^{0}-e^{-E_{R}} \partial u^{\prime} \Psi_{4}^{0}\right], \\
& \Psi_{2}^{\prime 0}=e^{-3 E_{R}}\left[\Psi_{2}^{0}-2 e^{-E_{R}} \partial u^{\prime} \Psi_{3}^{0}+\left(e^{-E_{R}} \partial u^{\prime}\right)^{2} \Psi_{4}^{0}\right],
\end{aligned}
$$

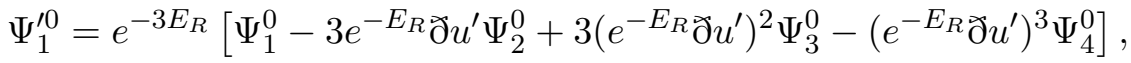

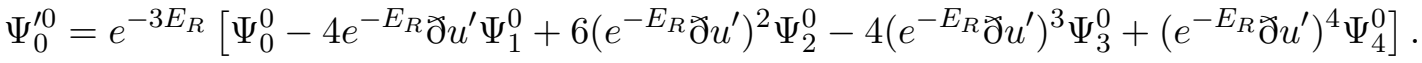

Open Access. This article is distributed under the terms of the Creative Commons Attribution License (CC-BY 4.0), which permits any use, distribution and reproduction in any medium, provided the original author(s) and source are credited. 


\section{References}

[1] Y.B. Zel'dovich and A.G. Polnarev, Radiation of gravitational waves by a cluster of superdense stars, Sov. Astron. 18 (1974) 17.

[2] D. Christodoulou, Nonlinear nature of gravitation and gravitational wave experiments, Phys. Rev. Lett. 67 (1991) 1486 [INSPIRE].

[3] V.B. Braginsky and L.P. Grishchuk, Kinematic Resonance and Memory Effect in Free Mass Gravitational Antennas, Sov. Phys. JETP 62 (1985) 427 [InSPIRE].

[4] V.B. Braginskii and K.S. Thorne, Gravitational-wave bursts with memory and experimental prospects, Nature 327 (1987) 123.

[5] A.G. Wiseman and C.M. Will, Christodoulou's nonlinear gravitational wave memory: Evaluation in the quadrupole approximation, Phys. Rev. D 44 (1991) R2945 [INSPIRE].

[6] K.S. Thorne, Gravitational-wave bursts with memory: The Christodoulou effect, Phys. Rev. D 45 (1992) 520 [INSPIRE].

[7] J. Frauendiener, Note on the memory effect, Class. Quant. Grav. 9 (1992) 1639.

[8] P.D. Lasky, E. Thrane, Y. Levin, J. Blackman and Y. Chen, Detecting gravitational-wave memory with LIGO: implications of GW150914, Phys. Rev. Lett. 117 (2016) 061102 [arXiv: 1605.01415] [INSPIRE].

[9] D.A. Nichols, Spin memory effect for compact binaries in the post-Newtonian approximation, Phys. Rev. D 95 (2017) 084048 [arXiv: 1702.03300] [InSPIRE].

[10] H. Yang and D. Martynov, Testing Gravitational Memory Generation with Compact Binary Mergers, Phys. Rev. Lett. 121 (2018) 071102 [arXiv:1803.02429] [INSPIRE].

[11] S. Weinberg, Infrared photons and gravitons, Phys. Rev. 140 (1965) B516 [INSPIRE].

[12] A. Strominger and A. Zhiboedov, Gravitational Memory, BMS Supertranslations and Soft Theorems, JHEP 01 (2016) 086 [arXiv:1411.5745] [INSPIRE].

[13] L. Bieri and D. Garfinkle, An electromagnetic analogue of gravitational wave memory, Class. Quant. Grav. 30 (2013) 195009 [arXiv: 1307.5098] [INSPIRE].

[14] S. Pasterski, Asymptotic Symmetries and Electromagnetic Memory, JHEP 09 (2017) 154 [arXiv: 1505.00716] [INSPIRE].

[15] L. Susskind, Electromagnetic Memory, arXiv: 1507.02584 [INSPIRE].

[16] P. Mao, H. Ouyang, J.-B. Wu and X. Wu, New electromagnetic memories and soft photon theorems, Phys. Rev. D 95 (2017) 125011 [arXiv:1703.06588] [INSPIRE].

[17] P. Mao and H. Ouyang, Note on soft theorems and memories in even dimensions, Phys. Lett. B 774 (2017) 715 [arXiv:1707.07118] [INSPIRE].

[18] M. Pate, A.-M. Raclariu and A. Strominger, Color Memory: A Yang-Mills Analog of Gravitational Wave Memory, Phys. Rev. Lett. 119 (2017) 261602 [arXiv:1707.08016] [INSPIRE].

[19] A. Ball, M. Pate, A.-M. Raclariu, A. Strominger and R. Venugopalan, Measuring Color Memory in a Color Glass Condensate at Electron-Ion Colliders, arXiv:1805.12224 [INSPIRE].

[20] H. Afshar, E. Esmaeili and M.M. Sheikh-Jabbari, String Memory Effect, JHEP 02 (2019) 053 [arXiv: 1811.07368] [INSPIRE]. 
[21] F. Cachazo and A. Strominger, Evidence for a New Soft Graviton Theorem, arXiv: 1404.4091 [INSPIRE].

[22] S. Pasterski, A. Strominger and A. Zhiboedov, New Gravitational Memories, JHEP 12 (2016) 053 [arXiv: 1502.06120] [INSPIRE].

[23] R. Penrose, Asymptotic properties of fields and space-times, Phys. Rev. Lett. 10 (1963) 66 [INSPIRE].

[24] R. Penrose, Zero rest mass fields including gravitation: Asymptotic behavior, Proc. Roy. Soc. Lond. A 284 (1965) 159 [INSPIRE].

[25] I. Robinson and A. Trautman, Spherical Gravitational Waves, Phys. Rev. Lett. 4 (1960) 431 [INSPIRE].

[26] L.P. Grishchuk and A.G. Polnarev, Gravitational wave pulses with 'velocity coded memory.', Sov. Phys. JETP 69 (1989) 653 [InSPIRE].

[27] J. Podolsky and R. Steinbauer, Geodesics in space-times with expanding impulsive gravitational waves, Phys. Rev. D 67 (2003) 064013 [gr-qc/0210007] [InSPIRE].

[28] J. Podolsky and R. Svarc, Refraction of geodesics by impulsive spherical gravitational waves in constant-curvature spacetimes with a cosmological constant, Phys. Rev. D 81 (2010) 124035 [arXiv: 1005.4613] [INSPIRE].

[29] J. Podolsky, C. Sämann, R. Steinbauer and R. Svarc, The global uniqueness and $C^{1}$-regularity of geodesics in expanding impulsive gravitational waves, Class. Quant. Grav. 33 (2016) 195010 [arXiv: 1602.05020] [INSPIRE].

[30] P.M. Zhang, C. Duval, G.W. Gibbons and P.A. Horvathy, The Memory Effect for Plane Gravitational Waves, Phys. Lett. B 772 (2017) 743 [arXiv: 1704.05997] [INSPIRE].

[31] P.M. Zhang, C. Duval and P.A. Horvathy, Memory Effect for Impulsive Gravitational Waves, Class. Quant. Grav. 35 (2018) 065011 [arXiv: 1709. 02299] [INSPIRE].

[32] P.M. Zhang, C. Duval, G.W. Gibbons and P.A. Horvathy, Velocity Memory Effect for Polarized Gravitational Waves, JCAP 05 (2018) 030 [arXiv:1802.09061] [INSPIRE].

[33] G. Compère, A. Fiorucci and R. Ruzziconi, Superboost transitions, refraction memory and super-Lorentz charge algebra, JHEP 11 (2018) 200 [arXiv:1810.00377] [INSPIRE].

[34] E.E. Flanagan, A.M. Grant, A.I. Harte and D.A. Nichols, Persistent gravitational wave observables: general framework, Phys. Rev. D 99 (2019) 084044 [arXiv:1901.00021] [INSPIRE].

[35] I.I. Shapiro, Fourth Test of General Relativity, Phys. Rev. Lett. 13 (1964) 789 [INSPIRE].

[36] M. Visser, B. Bassett and S. Liberati, Superluminal censorship, Nucl. Phys. Proc. Suppl. 88 (2000) 267 [gr-qc/9810026] [INSPIRE].

[37] M. Visser, B. Bassett and S. Liberati, Perturbative superluminal censorship and the null energy condition, AIP Conf. Proc. 493 (1999) 301 [gr-qc/9908023] [INSPIRE].

[38] R.D. Reasenberg et al., Viking relativity experiment: Verification of signal retardation by solar gravity, Astrophys. J. 234 (1979) L219 [INSPIRE].

[39] E. Newman and R. Penrose, An approach to gravitational radiation by a method of spin coefficients, J. Math. Phys. 3 (1962) 566 [InSPIRE]. 
[40] E.T. Newman and T.W.J. Unti, Behavior of Asymptotically Flat Empty Spaces, J. Math. Phys. 3 (1962) 891 [INSPIRE].

[41] G. Barnich and C. Troessaert, Finite BMS transformations, JHEP 03 (2016) 167 [arXiv: 1601.04090] [INSPIRE].

[42] R. Geroch, Asymptotic structure of space-time, in Asymptotic structure of space-time, P. Esposito and L. Witten ed., Plenum, New York, U.S.A., (1977), pp. 1-105.

[43] G. Barnich and C. Troessaert, Symmetries of asymptotically flat 4 dimensional spacetimes at null infinity revisited, Phys. Rev. Lett. 105 (2010) 111103 [arXiv:0909.2617] [INSPIRE].

[44] G. Barnich and C. Troessaert, Aspects of the BMS/CFT correspondence, JHEP 05 (2010) 062 [arXiv: 1001.1541 ] [INSPIRE].

[45] G. Barnich and C. Troessaert, Supertranslations call for superrotations, PoS (CNCFG2010) 010 (2010) [arXiv: 1102.4632] [INSPIRE].

[46] A. Strominger and A. Zhiboedov, Superrotations and Black Hole Pair Creation, Class. Quant. Grav. 34 (2017) 064002 [arXiv: 1610.00639] [INSPIRE].

[47] E.T. Newman and R. Penrose, Spin-coefficient formalism, Scholarpedia 4 (2009) 7445.

[48] S.W. Hawking and G.F.R. Ellis, The Large Scale Structure of Space-Time, Cambridge Monographs on Mathematical Physics, Cambridge University Press, (2011), [https://doi.org/10.1017/CBO9780511524646].

[49] E. Conde and P. Mao, BMS Supertranslations and Not So Soft Gravitons, JHEP 05 (2017) 060 [arXiv: 1612.08294] [INSPIRE].

[50] E.T. Newman and R. Penrose, 10 exact gravitationally-conserved quantities, Phys. Rev. Lett. 15 (1965) 231 [INSPIRE].

[51] E.T. Newman and R. Penrose, New conservation laws for zero rest-mass fields in asymptotically flat space-time, Proc. Roy. Soc. Lond. A 305 (1968) 175 [inSPIRE].

[52] G. Compère, R. Oliveri and A. Seraj, Gravitational multipole moments from Noether charges, JHEP 05 (2018) 054 [arXiv: 1711. 08806] [INSPIRE].

[53] H. Godazgar, M. Godazgar and C.N. Pope, Subleading BMS charges and fake news near null infinity, JHEP 01 (2019) 143 [arXiv:1809.09076] [INSPIRE].

[54] H. Godazgar, M. Godazgar and C.N. Pope, New dual gravitational charges, Phys. Rev. D 99 (2019) 024013 [arXiv: 1812.01641] [INSPIRE].

[55] H. Godazgar, M. Godazgar and C.N. Pope, Tower of subleading dual BMS charges, JHEP 03 (2019) 057 [arXiv: 1812.06935] [inSPIRE].

[56] G. Barnich, P. Mao and R. Ruzziconi, work in progress.

[57] S. Chandrasekhar, The Newman-Penrose formalism, in The mathematical theory of black holes, Clarendon Press, Oxford, U.K., (1983), pp. 40-55. 